\title{
Simultaneous Estimation of Indirect and Interaction Effects using Structural Equation Models
}

\author{
Joan Manuel Batista-Foguet ${ }^{1}$, Germà Coenders ${ }^{2}$, Willem E. Saris ${ }^{1}$, \\ and Josep Bisbe ${ }^{1}$
}

\begin{abstract}
Interaction effects are usually modeled by means of moderated regression analysis. Structural equation models with non-linear constraints make it possible to estimate interaction effects while correcting for measurement error. From the various specifications, Jöreskog and Yang's (1996, 1998), likely the most parsimonious, has been chosen and further simplified. Up to now, only direct effects have been specified, thus wasting much of the capability of the structural equation approach. This paper presents and discusses an extension of Jöreskog and Yang's specification that can handle direct, indirect and interaction effects simultaneously. The model is illustrated by a study of the effects of an interactive style of use of budgets on both company innovation and performance.
\end{abstract}

\section{Introduction}

Moderated regression analysis (MRA) - a particular specification of multiple linear regression analysis- has been widely used in management research for testing models that involve the presence of a variable that influences the impact of an independent variable on a dependent variable. In its usual formulation, MRA includes as an additional regressor a multiplicative term between two exogenous (independent) variables which represents the interaction effect (Jaccard et al., 1990; Aiken and West, 1991; Hartmann and Moers, 1999; Irwin and McClelland, 2001). Instances of recent applications of MRA in management research can be

\footnotetext{
${ }^{1}$ Department of Methods. ESADE. Ramon Llull University, Av. Pedralbes 60-62, E-080034 Barcelona (Spain); batista@esade.edu

${ }^{2}$ Department of Economics. University of Girona, FCEE, Montilivi Campus, E-17071 Girona (Spain); germa.coenders@udg.es
} 
found everywhere from Accounting (Li and Atuahene-Gima, 2001; Abernethy and Brownell, 1999), to Marketing (Sethi et al., 2001).

However, because of measurement error, the estimates of regression coefficients in MRA are not consistent. Biased estimates -actually attenuated estimates- limit the use of the technique to purely predictive purposes. This bias is especially relevant for interaction effects that are usually of low magnitude and may easily go undetected if attenuated. Additionally, the estimated standard errors of regression coefficients are also biased; so, no coherent inferences about population parameters or relationships among variables can be made.

The use of structural equations models (SEM) for correcting for measurement error has been proposed in the management literature, mainly by researchers in marketing (Bagozzi and Yi, 1989; Homer, 1990; Ashok et al., 2002). However, SEM have been proposed only rarely for estimating interaction effects in management (Ping, 1995). This paper critically addresses the problem of modeling and testing interaction hypotheses. It has three purposes:

- to contribute to the discussion among methodologists on SEM (Jöreskog and Yang, 1996; Ping, 1995, 1996; Jaccard and Wan, 1996; Li et al., 1998; Jöreskog, 1998; Algina and Moulder, 2001; Schumacker, 2002; Moulder and Algina, 2002) by combining aspects of the different strategies. We use centered indicators as Jackard and Wan (1996) propose for avoiding collinearity while using a single indicator for interaction as Jöreskog and Yang (1996) propose to increase parsimony.

- to offer an extension of the usual specification for modeling interaction effects. So far, interaction has only been specified among exogenous latent variables, ignoring the usual case of having indirect effects simultaneously. This leads to the specification of a simultaneous equation system.

- to use the full strength of SEM by distinguishing between direct, indirect and total effects, and discussing the interpretation of the interaction effects in the context of a simultaneous equation model.

The paper is organized as follows. First, current SEM approaches for modeling interactions are briefly reviewed. Second, a modification of the Jöreskog and Yang (1996) single indicator approach is discussed, and third, a simultaneous equation model is proposed as an extension of the product indicant approach (Kenny and Judd, 1984) and specifically as a generalization of the Jöreskog and Yang model. Discussion of testing and interpretation of the interaction effects follows. Finally, in order to illustrate this proposal, the new model specification is used to examine the potential direct, indirect and moderating effects of an interactive use of budgets as management control systems on both innovation and on performance of firms. 


\section{Approaches to model interactions with latent variables}

The constructs involved in management and marketing research, such as the ones just mentioned (i.e. innovation, performance, use of budgets) lead to models including latent variables which contain measurement error. So, instead of using observable regressors, researchers have tended to use multiple indicators to measure an underlying assumed continuous construct. Then the latent variable is specified by combining these indicators, frequently by simply adding their scores (Spector, 1992; Likert, 1932; Simpson, 1755) which does not completely solve the problem, as regressors obained with summated rating scales still contain measurement error.

Since the 1970s (Jöreskog, 1973) a major advantage of SEM is its capability to correct the estimates of the linear relationships between latent variables, both direct and indirect, for the measurement errors

In the 80's Kenny and Judd (1984) proposed a possible specification for modeling interaction effects under the SEM approach, which assumed that both interacting variables are continuous. Kenny and Judd's approach requires each latent variable to relate to at least two indicators and implies the formation of multiple indicators based on the products of the observed variables. These products are then used as indicators of the latent interaction. Different alternatives have been proposed for developing Kenny and Judd's approach. These include Jaccard and Wan's (1995) multiple product indicators approach, Jöreskog and Yang's (1996) single product indicator approach, Ping's (1995, 1996) two-step single and multiple product indicators approach, and Bollen and Paxton (1998) two-stage least squares single indicator multiple instrumental variables approach. When one or both of the interacting variables are discrete and the number of cases in each class large enough, then a "multigroup" approach should be applied (e.g., Batista-Foguet et al., 2001; Lomax, 1983).

Most of these product indicant approaches require SEM programs that permit the use of non-linear constraints. Developments in software and contributions by Jöreskog and Yang (1996) and Ping (1995) have made it much easier to apply the Kenny and Judd approach, highlighting problems and issues related to modeling interaction effects in latent variable models.

\subsection{Ways to obtain the indicators of a latent interaction in SEM}

It is not the aim of this paper to provide a comprehensive presentation of the various procedures currently available for testing interaction effects in structural equation modeling (See Li et al., 1998; or Schumacker and Marcoulides, 1998). 
The Jaccard and Wan (1995) multiple indicator method simultaneously includes all cross-products of the original indicators of both interacting latent variables. So it is a straightforward but not parsimonious implementation of Kenny and Judd's approach. In addition, this approach leads to problems of nonnormality of the indicators because the product of two normally distributed variables is not itself normally distributed even if the original variables are.

Jöreskog and Yang (1996) argued that multiple indicators for the interaction are not necessary; pointing out that the model can be identified with a single indicator of the product variable, which leads to a model with fewer parameters, fewer complex constraints and fewer non-normal product indicators.

Ping $(1995,1996)$ uses summated rating scales to compute scores for both interacting latent variables, and then multiplies these scales to obtain a single indicator of the interaction. The author derives the expressions of the loading and the error variance of the interaction indicator based on those of the original indicators, which can be used in two alternative ways. In a more convenient twostep strategy to fix the values of the measurement parameters of the full model, using estimates obtained in a previous confirmatory factor analysis of the indicators of both latent variables that interact. In a more sophisticated one-step strategy as a set of constraints involving the measurement parameters in the estimation of the full model. The performance of the standard errors and test statistics in the former two-step strategy has not been studied.

This paper focuses on the approach by Jöreskog and Yang (1996), and developments by Yang Jonsson (1998) and Yang Wallentin and Jöreskog (2001). These authors use non-centered indicators because they consider the means of the latent variables, which are related to the means of the observed indicators and to other model parameters. However, this consideration implies additional constraints in the structure of the measurement model. These constraints can be avoided if the purpose of the analysis refers only to the covariances among latent variables. We therefore suggest modifying Jöreskog and Yang's approach by using centered indicators and modelling only covariances, which brings the approach closer to those of Jackard and Wan and of Ping. In addition, centered indicators would avoid collinearity with the latent interaction, a point which we take up later.

\section{Jöreskog and Yang's specification in SEM for modeling interaction effects. Single equation model}

In general the following model has been specified for the latent variables, represented by the Greek letter $\eta$ as opposed to the observed variables: 


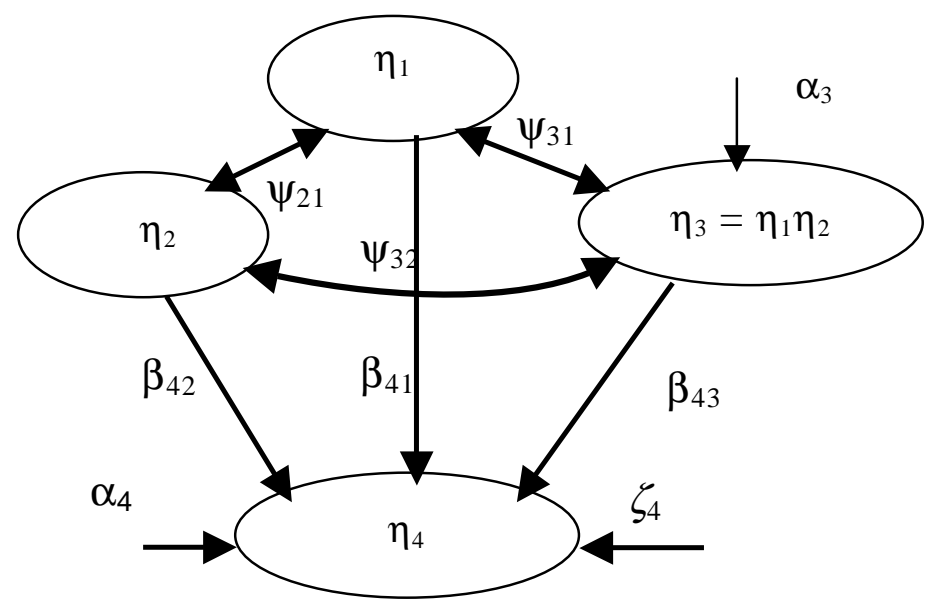

Figure 1: Single equation SEM for modeling interaction effects.

This latent exogenous variables model can be presented by the following equation:

$$
\begin{aligned}
& \eta_{4}=\alpha_{4}+\beta_{41} \eta_{1}+\beta_{42} \eta_{2}+\beta_{43} \eta_{3}+\zeta_{4} \\
& \text { where } \eta_{3}=\eta_{1} \cdot \eta_{2}
\end{aligned}
$$

where $\beta_{4 k}$ stands for the regression coefficient of $\eta_{4}$ on $\eta_{k}$ and $\zeta_{4}$ is the disturbance term. In this approach all three variables $\eta_{1}, \eta_{2}$ and $\eta_{3}$ are exogenous variables with free variances $\psi_{k k}$ and covariances $\psi_{k k}$. Although $\eta_{1}$ and $\eta_{2}$ are centered, $\eta_{3}$ is not. So, other parameters are $\mathrm{E}\left(\eta_{3}\right)=\alpha_{3}$ and $\operatorname{Var}\left(\zeta_{4}\right)=\psi_{44}$.

It is assumed, without loss of generality, that the first exogenous latent variable has two indicators, the second three indicators, and the endogenous latent variable (whose measurement error does not lead to coefficient bias) one indicator. As is usual (Jöreskog and Yang, 1996; Yang Jonsson, 1998) the first indicator of each variable is used to build the single indicator of the interaction $\left(y_{6}=y_{1} y_{3}\right)$. These assumptions lead to a measurement model that can be specified as follows:

$$
\left\{\begin{array}{l}
y_{1}=\tau_{1}+\lambda_{11} \eta_{1}+\varepsilon_{1} \\
y_{2}=\tau_{2}+\lambda_{21} \eta_{1}+\varepsilon_{2} \\
y_{3}=\tau_{3}+\lambda_{32} \eta_{2}+\varepsilon_{3} \\
y_{4}=\tau_{4}+\lambda_{42} \eta_{2}+\varepsilon_{4} \\
y_{5}=\tau_{5}+\lambda_{52} \eta_{2}+\varepsilon_{5} \\
y_{6}=\tau_{6}+\lambda_{61} \eta_{1}+\lambda_{62} \eta_{2}+\lambda_{63} \eta_{3}+\varepsilon_{6} \\
y_{7}=\eta_{4}
\end{array}\right.
$$

For identification purposes, and without any loss of generality, the scale of the latent variables is fixed by constraining the loadings of the indicators that are used to compute the product indicator: 


$$
\lambda_{11}=1 \text { and } \lambda_{32}=1
$$

Additional parameters of the measurement part are $\operatorname{Var}\left(\varepsilon_{j}\right)=\theta_{j j}$.

The specification is completed with the assumptions that $\eta_{1}, \eta_{2}$, and $\varepsilon_{1}$ to $\varepsilon_{5}$ are multivariate normally distributed with zero expectation. Additionally, $\varepsilon_{1}$ to $\varepsilon_{5}$ are assumed to be mutually independent (not only uncorrelated) and independent of $\eta_{1}$, $\eta_{2}$ and $\zeta_{4}$, and $\zeta_{4}$ independent of $\eta_{1}$ and $\eta_{2}$.

These assumptions allow us to decompose the expectation, variance and covariance of the product indicator, as well as to derive non-linear constraints, relating its associated parameters. Thus, the addition of the product indicant variable involves the estimation of very few additional free parameters.

If $y_{1}$ and $y_{3}$ are used to fix the scale of the latent variables, the single product term, $y_{6}$, is computed as:

$$
\begin{gathered}
\begin{array}{c}
y_{6}=y_{1} y_{3}=\left(\tau_{1}+\eta_{1}+\varepsilon_{1}\right)\left(\tau_{3}+\eta_{2}+\varepsilon_{3}\right) \\
=\tau_{1} \tau_{3}+\tau_{3} \eta_{1}+\tau_{1} \eta_{2}+\eta_{2} \eta_{1}+\varepsilon_{6}
\end{array} \\
\text { where } \varepsilon_{6}=\tau_{3} \varepsilon_{1}+\tau_{1} \varepsilon_{3}+\eta_{1} \varepsilon_{3}+\eta_{2} \varepsilon_{1}+\varepsilon_{1} \varepsilon_{3}
\end{gathered}
$$

The following constraints can be derived from the expressions of $y_{6}$ in Equations 3.3 and 3.5:

$$
\left\{\begin{array}{l}
\tau_{6}=\tau_{1} \tau_{3} \\
\lambda_{61}=\tau_{3} \\
\lambda_{62}=\tau_{1} \\
\lambda_{63}=1
\end{array}\right.
$$

The measurement error variances and covariances can be derived from Equation 3.6, which involves the following constraints:

$$
\begin{aligned}
& \left\{\begin{array}{l}
\theta_{61}=\mathrm{E}\left(\varepsilon_{6} \varepsilon_{1}\right)=\tau_{3} \mathrm{E}\left(\varepsilon_{1} \varepsilon_{1}\right)=\tau_{3} \theta_{11} \\
\theta_{63}=\mathrm{E}\left(\varepsilon_{6} \varepsilon_{3}\right)=\tau_{1} \mathrm{E}\left(\varepsilon_{3} \varepsilon_{3}\right)=\tau_{1} \theta_{33}
\end{array}\right. \\
& \begin{aligned}
\theta_{66}= & \operatorname{Var}\left(\varepsilon_{6}\right)=\operatorname{Var}\left(\tau_{3} \varepsilon_{1}+\tau_{1} \varepsilon_{3}+\eta_{1} \varepsilon_{3}+\eta_{2} \varepsilon_{1}+\varepsilon_{1} \varepsilon_{3}\right) \\
= & \tau_{3}^{2} \operatorname{Var}\left(\varepsilon_{1}\right)+\tau_{1}^{2} \operatorname{Var}\left(\varepsilon_{3}\right)+\operatorname{Var}\left(\eta_{1}\right) \operatorname{Var}\left(\varepsilon_{3}\right)+\operatorname{Var}\left(\eta_{2}\right) \operatorname{Var}\left(\varepsilon_{1}\right) \\
& +\operatorname{Var}\left(\varepsilon_{1}\right) \operatorname{Var}\left(\varepsilon_{3}\right) \\
= & \tau_{3}^{2} \theta_{11}+\tau_{1}^{2} \theta_{33}+\psi_{11} \theta_{33}+\psi_{22} \theta_{11}+\theta_{11} \theta_{33}
\end{aligned}
\end{aligned}
$$

Under bivariate normality of the main effects $\eta_{1}$ and $\eta_{2}$ (Anderson, 1984), the variance of the product of latent variables is:

$$
\psi_{33}=\operatorname{Var}\left(\eta_{3}\right)=\operatorname{Var}\left(\eta_{1} \cdot \eta_{2}\right)=\operatorname{Var}\left(\eta_{1}\right) \operatorname{Var}\left(\eta_{2}\right)+\operatorname{Cov}^{2}\left(\eta_{1} \eta_{2}\right)=\psi_{11} \psi_{22}+\psi_{21}^{2}
$$


And its expectation:

$$
\alpha_{3}=E\left(\eta_{3}\right)=E\left(\eta_{1} \eta_{2}\right)=\operatorname{Cov}\left(\eta_{1} \eta_{2}\right)=\psi_{21}
$$

Although this model leads to some extra parameters $\left(\lambda_{61}, \lambda_{62}, \lambda_{63}, \psi_{33}, \theta_{61}, \theta_{63}\right.$, $\theta_{66}, \tau_{6}$ and $\alpha_{3}$ ), it has been shown above that all of them can be expressed as functions of other parameters of the model, so that using these constraints the only extra parameters to be estimated are $\psi_{31}, \psi_{32}$ (that is, the covariances of latent variables that are customarily free and identified) and $\beta_{43}$, the parameter of interest. So with a litle extra effort, one can specify these constraints and this model can be estimated. Constraint 3.10 (requiring normality) is not needed for identification and can be omitted if $\eta_{1}$ and $\eta_{2}$ are non-normal.

\section{Modification of the Jöreskog and Yang approach to model interactions}

\subsection{Use of centered indicators}

It has to be noted that any indicant product approach may lead to substantial collinearity, precisely because the measures associated with the interaction construct are functions of the measures of the main effect constructs (Ridgeon et al., 1998). Consequently, in order to avoid collinearity, we suggest that the analysis is conducted on centered indicators of the interacting variables (See Li et al., 1998; and the Appendix in Irwin and McClelland, 2001, for a more detailed discussion on the elimination of collinearity by changing the origin of the variables which interact). This involves computing $y_{6}$ from the centered indicators $y_{1}$ and $y_{3}$ and subsequently centering $y_{6}$ again.

In those cases where the mean structure parameters $\alpha$ and $\tau$ are not of interest to the researcher, Equations 3.1 and 3.3 become shorter and constraints in Equations 3.7 to 3.11 from Jöreskog and Yang's approach can be reduced dramatically in both number and complexity, thus simplifying the analysis while preventing collinearity. Since for every original centered indicator the measurement intercept, $\tau$, is zero, and the expectations and intercepts $\alpha$ of all latent variables are also zero (even $\eta_{3}$ is implicitly redefined as $\eta_{3}-\alpha_{3}$ ), these equations and constraints become:

$$
\eta_{4}=\beta_{41} \eta_{1}+\beta_{42} \eta_{2}+\beta_{43} \eta_{3}+\zeta_{4}
$$




$$
\begin{aligned}
& \qquad\left\{\begin{array}{l}
y_{1}=\eta_{1}+\varepsilon_{1} \\
y_{2}=\lambda_{21} \eta_{1}+\varepsilon_{2} \\
y_{3}=\eta_{2}+\varepsilon_{3} \\
y_{4}=\lambda_{42} \eta_{2}+\varepsilon_{4} \\
y_{5}=\lambda_{52} \eta_{2}+\varepsilon_{5} \\
y_{6}=\eta_{3}+\varepsilon_{6} \\
y_{7}=\eta_{4}
\end{array}\right. \\
& y_{6}=y_{1} y_{3}=\eta_{2} \eta_{1}+\varepsilon_{6}
\end{aligned}
$$

which leads only to the following simple constraints:

$$
\begin{aligned}
& \theta_{66}=\psi_{11} \theta_{33}+\psi_{22} \theta_{11}+\theta_{11} \theta_{33} \\
& \psi_{33}=\psi_{11} \psi_{22}+\psi_{21}^{2}
\end{aligned}
$$

with $\lambda_{63}=1$ and $\tau_{6}=\lambda_{61}=\lambda_{62}=\theta_{61}=\theta_{63}=0$.

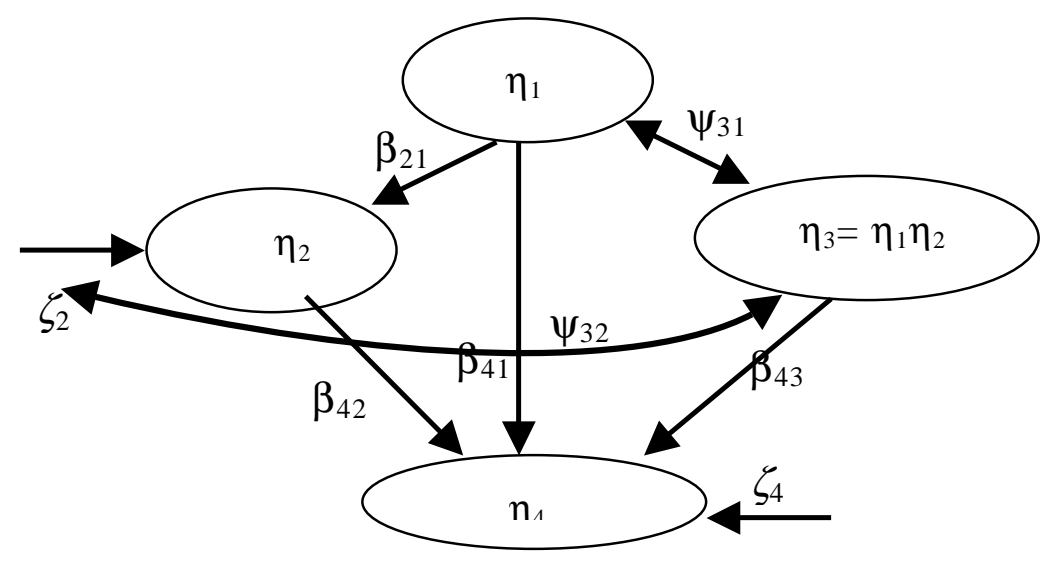

Figure 2: Structural equation model including direct, indirect and interaction effects simultaneously.

\subsection{A simultaneous equation system. Specification}

Interactions with SEM have so far only been modeled with one equation, where the regressors that interact are exogenous latent variables (Schumacker and Marcoulides, 1998; Li et al., 1998; Schumacker, 2002; Moulder and Algina, 2002). This single equation formulation discussed in the SEM literature and presented in the previous section allows for correction of measurement error but, due to the use of a single equation, it only makes it possible to estimate the interaction effect. In order to 
also estimate indirect effects in the same model, a simultaneous equation system must be specified which extends the usual single indicator specification by Yang and Jöreskog (1996), including direct, indirect and moderating effects in the same model. The structural part of our proposal is specified in Figure 2.

The structural part of the model includes Equation $4.1 \mathrm{~b}$ and an additional equation for the relationship among the up to now exogenous variables, $\eta_{1}$ and $\eta_{2}$.

$$
\eta_{2}=\beta_{21} \eta_{1}+\zeta_{2}
$$

Now only $\eta_{1}$ and $\eta_{3}=\eta_{1} \eta_{2}$ remain exogenous. Additional parameters of the structural part are $\operatorname{Var}\left(\eta_{k}\right)=\psi_{k k} ; k=1$ to 4 . As in the one-equation specification, all covariances between $\eta_{3}$ and its constituents $\eta_{1}$ and $\eta_{2}$ are model parameters: $\operatorname{Cov}\left(\eta_{3,} \eta_{1}\right)=\psi_{13}$ and $\operatorname{Cov}\left(\eta_{3}, \zeta_{2}\right)=\psi_{32}$. Unlike the previous case, now $\psi_{32}$ involves a disturbance term.

The main difference with the previous specification is that not all the variances and covariances of $\eta_{1}$ and $\eta_{2}$ are model parameters but rather functions of model parameters that can easily be derived from path analysis or from variance and covariance algebra:

$$
\left\{\begin{array}{l}
\operatorname{Var}\left(\eta_{2}\right)=\psi_{22}+\beta^{2}{ }_{21} \psi_{11} \\
\operatorname{Cov}\left(\eta_{1}, \eta_{2}\right)=\beta_{21} \psi_{11}
\end{array}\right.
$$

The measurement part is specified as before in Equation 4.3b. The specification is completed with the assumptions that $\eta_{1}, \zeta_{2}, \eta_{3}, \zeta_{4}$ and $\varepsilon_{1}$ to $\varepsilon_{5}$ are multivariate normally distributed with zero expectation. In addition, the $\varepsilon$ terms are independent, both mutually and with all $\eta$ and $\zeta_{\text {. }} \zeta_{4}$ is independent of $\eta_{3}, \zeta_{2}$ and $\eta_{1}$ and $\zeta_{2}$ is independent of $\eta_{1}$.

Substituting in Equation $4.9 \mathrm{~b}$ the new expression for the variance of $\eta_{2}$ yields:

$$
\theta_{66}=\psi_{11} \theta_{33}+\left(\beta_{21}^{2} \psi_{11}+\psi_{22}\right) \theta_{11}+\theta_{11} \theta_{3}
$$

Finally, the variance of the product of the latent variables is:

$$
\begin{aligned}
\psi_{33}= & \operatorname{Var}\left(\eta_{1}\right) \operatorname{Var}\left(\eta_{2}\right)+\operatorname{Cov}^{2}\left(\eta_{1} \eta_{2}\right)= \\
& \psi_{11}\left(\psi_{22}+\beta_{21}^{2} \psi_{11}\right)+\left(\beta_{21} \psi_{11}\right)^{2}=\psi_{11} \psi_{22}+2 \beta_{21}^{2} \psi_{11}^{2}
\end{aligned}
$$

Given that the model in this section is saturated with respect to the relationships for the latent variables and thus is equivalent to the previous model, it leads to the same results for Equations $4.1 \mathrm{~b}$ and $4.3 \mathrm{~b}$. However, the model in this section also estimates the relationship between $\eta_{1}$ and $\eta_{2}$ and thus both a direct and an indirect effect from $\eta_{1}$ to $\eta_{4}$.

We have chosen the simplest model with only two equations where one interacting variable has a causal effect on the other variable. However the same 
approach could also be used if one or more exogenous variables would have an effect on $\eta_{1}$ and $\eta_{2}$. The formulation would remain the same, only a substitute for Equation 4.13 would have to be derived by path analysis, including $\operatorname{Var}\left(\eta_{1}\right)$ if needed, which would have obvious implications for Equations $4.9 \mathrm{c}$ and $4.10 \mathrm{c}$.

\subsection{Interpretation}

The effect of $\eta_{2}$ on $\eta_{4}$ conditional on different values of $\eta_{1}$, can be obtained from the expected value in the expression

$$
\mathrm{E}\left(\eta_{4}\right)=\beta_{41} \eta_{1}+\beta_{42} \eta_{2}+\beta_{43} \eta_{1} \eta_{2}
$$

as the partial derivative $\mathrm{E}\left(\eta_{4}\right)$ with respect to $\eta_{2}$.

$$
\frac{\partial \mathrm{E}\left(\eta_{4}\right)}{\partial \eta_{2}}=\beta_{42}+\beta_{43} \eta_{1}
$$

Besides a direct main effect, this equation also displays a typical interaction effect, in which the effect of $\eta_{2}$ on $\eta_{4}$ depends on the value of $\eta_{1}$. Thus, the interpretation of the main effect $\beta_{42}$ parameter is that occurring when the value of the other variable is zero. If $\eta_{1}$ and $\eta_{2}$ are mean centered, $\beta_{42}$ can be more easily interpreted as the effect for the mean value of the other variable.

In order to assess the effect of $\eta_{1}$, its relationship to $\eta_{2}$ has to be taken into account, so that:

$$
\frac{\partial \mathrm{E}\left(\eta_{2}\right)}{\partial \eta_{1}}=\beta_{21}
$$

And the partial derivative of Equation 4.14, now with respect to $\eta_{1}$ gives us the total effect of $\eta_{1}$ on $\eta_{4}$ as:

$$
\frac{\partial \mathrm{E}\left(\eta_{4}\right)}{\partial \eta_{1}}=\beta_{41}+\beta_{42} \beta_{21}+\beta_{43}\left(\eta_{2}+\beta_{21} \eta_{1}\right)
$$

This equation displays a direct main effect $\beta_{41}$, an indirect effect $\beta_{42} \beta_{21}$, the interaction effect $\beta_{43} \eta_{2}$ and a combined interaction-indirect effect $\beta_{43} \beta_{21} \eta_{1}$. Thus, the effect of $\eta_{1}$ on $\eta_{4}$ depends on the value of $\eta_{2}$ to a greater extent than one should expect from the interaction effect alone. If we omit all terms related to the indirect effect we have the direct main and interaction effects as:

$$
\beta_{41}+\beta_{43} \eta_{2}
$$


Standardization prevents $\eta_{3}$ from being equal to $\eta_{1} \eta_{2}$ and thus Equation 4.14 from holding (see Jaccard et al., 1990). Thus, all interpretations in this section must never be done with standardized parameters.

\subsection{Estimation and testing}

In general, for SEM normality is not required for consistency of ML estimates (e.g. Satorra, 1990), but only for the correctness of standard errors and test statistics. However, in the interaction model case, the restriction in equation 4.10c only holds under normality of the main effects, and thus, non-normality implies a specification error and can lead to bias. The fulfillment of the normality assumption must thus be thoroughly assessed prior to fitting this type of models. If the main effect indicators appear to be non normal, then the model should be fitted with constraint $9 \mathrm{c}$ only.

However, since the product indicant will not be normally distributed even if the main effect indicators are, methods that compute standard errors and test statistics that are robust to departures from normality are required even in the normal case (Yang-Wallentin and Jöreskog, 2001).

Robust procedures in SEM appeared earlier on. Browne (1984) suggested an alternative asymptotically distribution free estimation method that later research has reported to be limited in all but extremely large samples (e.g. Muthén and Kaplan, 1992; Fouladi, 2000).

Following a different path, Satorra and Bentler $(1988,1994)$ developed robust test statistics such as the mean-and-variance adjusted $\chi^{2}$ statistic, the mean scaled $\chi^{2}$ statistic and robust standard errors. These statistics are the right ones to use under arbitrary distributions when using the still consistent standard ML estimation method. Among these robust statistics, the mean scaled $\chi^{2}$ statistic is preferred for smaller samples (Satorra, 2001).

On the contrary, t-tests of significance of individual parameters like the interaction term using robust standard errors are not recommended for small samples (Boomsma and Hoogland, 2001). Mean-scaled $\chi^{2}$ differences should be used instead. Unfortunately, the difference between two mean-scaled $\chi^{2}$ statistics is not $\chi^{2}$ distributed, though some easy adjustments can be made by hand (Satorra and Bentler, 2001).

Let $T^{*}{ }_{0}$ and $T^{*}{ }_{1}$ be the mean-scaled $\chi^{2}$ statistics, $T_{0}$ and $T_{1}$ the standard ML $\chi^{2}$ statistics, $c_{0}$ and $c_{1}$ the scaling constants obtained as $c_{0}=T_{0} / T^{*}{ }_{0}$ and $c_{1}=T_{1} / T^{*}{ }_{1}$, and $d_{0}$ and $d_{l}$ the degrees of freedom for two nested models, of which Model 0 is more restrictive. The robust $\chi^{2}$ difference can be computed as: 


$$
\text { Robust } \chi^{2} \text { difference }=\frac{T_{0}-T_{1}}{\frac{d_{0} c_{0}-d_{1} c_{1}}{\left(d_{0}-d_{1}\right)}}
$$

The LISREL8.5 program (Du Toit and Du Toit, 2001) includes these robust statistics and allows the researcher to introduce non-linear constraints and is thus appropriate for the estimation of this model.

\section{An illustration}

\subsection{Background}

The example we will use comes from a study by Bisbe (2002). He analyzes the effects of the style of use of budgets $\left(\eta_{1}\right)$ on innovation $\left(\eta_{2}\right)$ and performance $\left(\eta_{4}\right)$. In Bisbe's study, following Simon's framework (Simons, 1991, 1995, 2000) the following hypotheses are formulated:

- Hypothesis 1: it is postulated that the more interactive the use of budgets, the greater the innovation. This is equivalent to saying that $\beta_{21}$ is larger than zero.

- Hypothesis 2: it is postulated that, the greater the innovation, the better the performance. This hypothesis can be considered to hold if Equation 4.15 is greater than zero for all values in the usual range of variation of $\eta_{1}$. Hypotheses 1 and 2 together imply an indirect effect of interactive use of budgets on performance.

- Hypothesis 3: it is expected that the interactive use of budgets has a larger effect on performance when innovation is high, which leads to the inclusion of an interaction term $\eta_{3}$, and to the effect $\beta_{43}$ being positive.

- Hypothesis 4: there may also be a direct effect of the interactive use of budgets on performance. This is equivalent to saying that Equation 4.18 is larger than zero for all values in the usual range of variation of $\eta_{2}$. Even if this hypothesis does not hold, $\beta_{41}$ must also be included in the model as the inclusion of interactions makes all main effects necessary in order not to get misleading interaction estimates (Irwin and McClelland, 2001).

All these hypotheses make our model identical to that of Figure 2. Note that some of them are directly related to values of a single model parameter while others are not. 


\subsection{Data and measurement instruments}

Bisbe (2002) developed multi-item instruments for measuring the following constructs:

Interactive Style-of-use of Budgets $\left(\eta_{1}\right)$ : $y_{1}$ : the extent to which information from the budget system demands frequent and regular attention from the top manager;

$y_{2}$ : the degree to which information from the budget system is discussed faceto-face on a permanent versus merely on an exception basis.

Product Innovation $\left(\eta_{2}\right)$ :

$y_{3}$ : part of the product portfolio corresponding to recently launched products;

$y_{4}$ : rate of introduction of new products;

$y_{5}$ : tendency of firms to pioneer.

These five items were in a Likert format ranging from 1 to 7 . In those cases where budgets were not used at all in the company, the items $\mathrm{y}_{1}$ and $\mathrm{y}_{2}$ were scored zero. The three innovation items, referred to the last three years as compared to industry average.

Performance $\left(\eta_{4}\right)$ : Based on the multidimensional self-rating instrument developed by Govindarajan $(1984,1988)$, performance was assessed through a battery of items that represent effectiveness on a series of financial (sales growth rate, revenue growth rate, return on investment, profit/sales ratio) and customer dimensions (customer satisfaction, customer retention, customer acquisition and increase in market share) over the last three years as compared with the industry average. Items were weighted according to their varying perceived relative importance. Composite scales are not free from measurement error, but this does not introduce any parameter bias when the variable measured with error does not have any effect on any other variable.

Data were gathered through the administration of a written questionnaire to a sample of chief executive officers of medium-sized, mature manufacturing firms with headquarters located in Catalonia, Spain (Bisbe, 2002). The whole population studied comprised 120 firms. 58 questionnaires were returned, all of which were complete. Thus, the response rate was $48.33 \%$. However, for the sake of consistency in the time framework of the study, cases where the executives reported less than three years in their current job position $(n=18)$ were excluded. The resulting useable sample was thus $n=40$.

Even though a sample size of 40 can be considered rather small, in SEM the precision and statistical properties of the estimates and the power of the tests do not only depend on sample size but also on a number of model characteristics. In regression models, parsimony, absence of collinearity and high percentages of explained variance reduce the sample sizes needed. In SEM, the list is completed 
with analogous characteristics referring to the measurement part: low numbers of latent variables, high numbers of indicators per latent variable, low number of free parameters, and high reliabilities of the indicators can reduce sample size requirements (e.g. Saris and Satorra, 1988).

\section{Results}

\subsection{Measurement model}

First, we specified a three-factor confirmatory factor analysis (CFA) model where the latent interaction has a single indicator as in Jöreskog and Yang (1996). The introduction of the latent interaction in the measurement model involves the introduction of the constraints in Equations $4.9 \mathrm{~b}$ and $4.10 \mathrm{~b}$. Additionally, the parsimony of the model was increased by assuming that the units of measurement were the same for all indicators of the same factor (Equation 6.1). As mentioned earlier, parsimony and high indicator reliability are going to be crucial with our small sample size. The estimates and path diagram of the model including Equation 6.1 are in Figure 3.

$$
\lambda_{21}=1, \lambda_{32}=1 \text { and } \lambda_{52}=1
$$

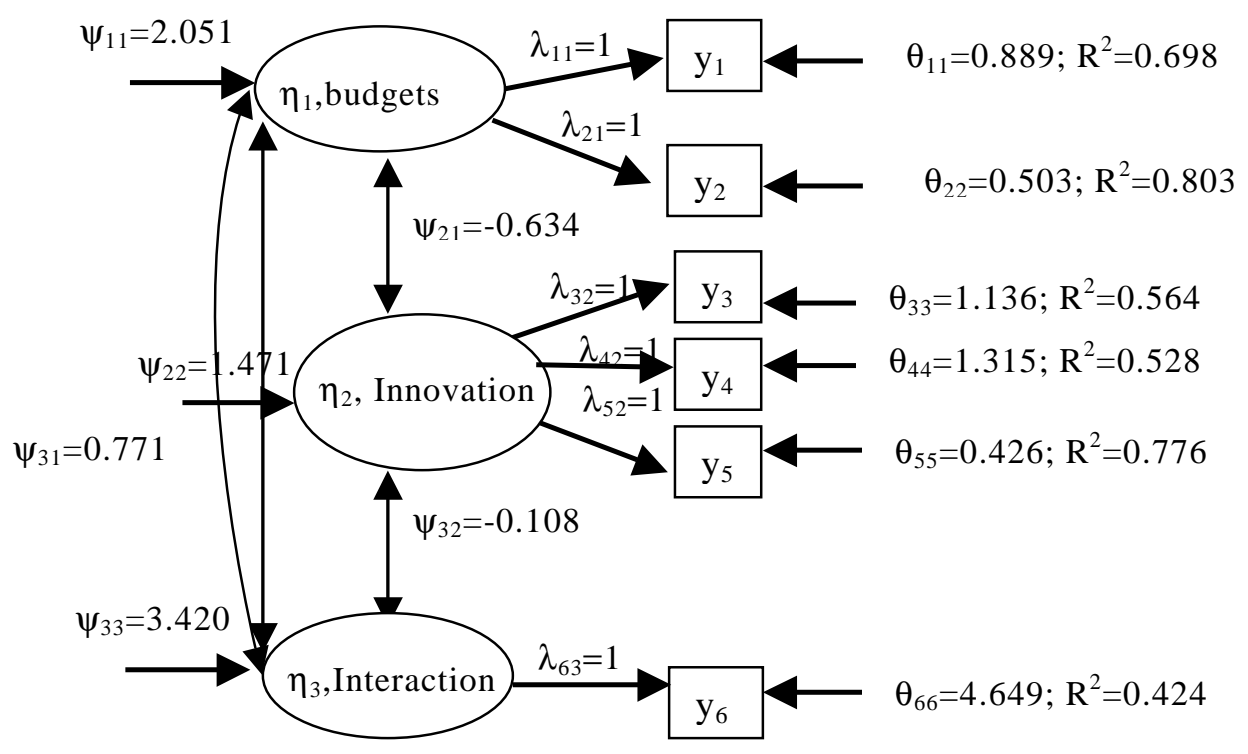

Figure 3: Estimates of the measurement model. 
The robust $\chi^{2}$ difference test statistic for the constraints in Equation 6.1 was 0.38 , with three degrees of freedom and is thus clearly non-significant ( $\mathrm{p}$ value $=0.94)$. The removal of the constraints would increase standard errors by $54 \%$, on average, over all parameters. This is comparable to the increase in standard errors that takes place if the sample size is divided by about two and a half. The high estimates of the reliabilities of the indicators and the low estimates of factor correlations ( 0.37 was the highest value in absolute terms) of the constrained model also help reduce small sample related problems.

The goodness of fit of the model with the constraints in Equation 6.1 was excellent with a Satorra-Bentler scaled $\chi^{2}$, of 14.602 with 11 degrees of freedom (p-value=0.201).

\subsection{Structural model}

The measurement and structural part are, of course, estimated simultaneously (See in the appendix the setup for modeling the interaction with LISREL 8.51) but since measurement estimates have already been presented in Figure 3 we only give the results of the structural part here, which is shown in the path diagram of Figure 2 .

Table 1: Estimates of the structural model ${ }^{1}$.

\begin{tabular}{|c|c|c|c|}
\hline $\begin{array}{l}\text { Parameter / } \\
\text { Index }\end{array}$ & Estimate & $\begin{array}{l}\text { Robust } \chi^{2} \\
\text { diference }\end{array}$ & $\begin{array}{l}\text { Robust } \\
\text { p-value }\end{array}$ \\
\hline$\beta_{43}$ & 0.306 & 5.89 & 0.015 \\
\hline$\beta_{42}$ & 0.415 & 2,19 & 0.139 \\
\hline$\beta_{41}$ & 0.073 & 0.24 & 0.624 \\
\hline$\beta_{21}$ & -0.309 & 3.63 & 0.057 \\
\hline $\mathrm{R}^{2}\left(\eta_{2}\right)$ & 0.132 & & \\
\hline $\mathrm{R}^{2}\left(\eta_{4}\right)$ & 0.552 & & \\
\hline robust $\chi^{2}$ & 15.80 & & \\
\hline d.f. & 14 & & \\
\hline $\mathrm{p}$-value & 0.326 & & \\
\hline
\end{tabular}

For testing the effect of the latent interaction on performance (constraint $\beta_{43}=0$ ) we used the robust $\chi^{2}$ difference statistic. The statistic is 5.89, with 1 degree of freedom and is thus clearly significant ( $\mathrm{p}$-value $=0.015$ ). The relevance of the interaction effect is also revealed by the fact that the $R^{2}$ of the equation of $\eta_{4}$ would drop from 0.552 to 0.286 if the interaction effect was removed. Hypothesis 3 is thus clearly supported. 
No other effects are significant. However, we can argue that the significance of the $\beta_{43}$ interaction effect implies that the $\beta_{42}$ and $\beta_{41}$ main effects must also be in the model even if non-significant (Irwin and McClelland, 2001). Hypothesis 1 is clearly not supported, while Hypotheses 2 and 4 involving main effects are assessed next.

The interpretation of the interaction effect follows naturally. A statistically significant interaction indicates that there is a non-additive effect (i.e. independent and proportional effects) of both factors on performance but rather that the effect of each factor depends on the value of the other. In other words, the expected effect of a factor should be interpreted conditional on the value of the other moderating factor.

For instance, Figure 4 illustrates how the effect of innovation on performance depends on the value of the interactive style-of-use of budgets, by showing the decomposition of the total effects of both factors on performance for the values 0 and 1 of the other factor.

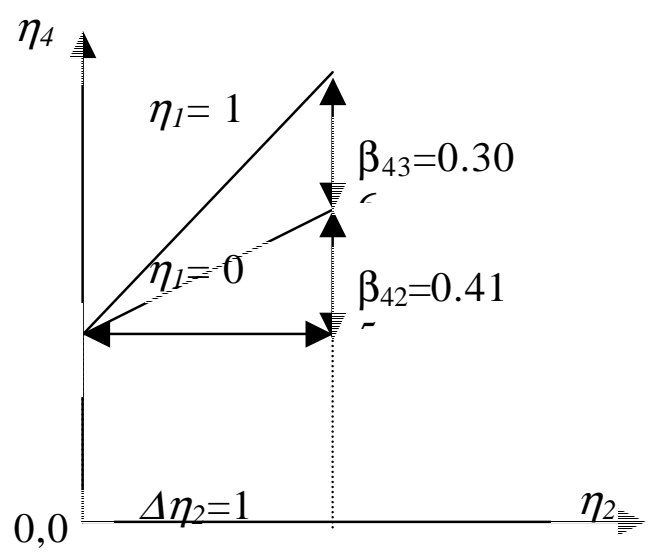

Figure 4: Decomposition of the total effect of innovation on performance.

Figure 4 illustrates the interpretation of the unstandardized total effect of $\eta_{2}$ on $\eta_{4}$, according to Equation 4.15 , as the direct effect $\left(\beta_{42}\right)$ plus the interaction effect $\left(\beta_{41} \eta_{1}\right)$. Thus, each additional unit of innovation raises the expected increase in performance by 0.721 if the interactive use of budgets equals 1 .

Thus, to assess hypotheses 2 and 4, which relate to the main effects of $\eta_{1}$ and $\eta_{2}$ on performance, these main effects have to be computed according to Equations 4.15 and 4.18 and must be evaluated conditional to the range of plausible values of the other factor. To represent this range, we took the percentiles 5, 25, 50, 75 and 95 of the items $y_{1}$ and $y_{3}$ that are used to fix the scale of $\eta_{1}$ and $\eta_{2}$ respectively. 
Table 2: Conditional effects on performance.

\begin{tabular}{|c|c|c|c|c|c|}
\hline percentile & 5 & 25 & 50 & 75 & 95 \\
\hline conditioned on $\eta_{l}=$ & - & - & - & 1.77 & 2.70 \\
\hline & 2.23 & 1.23 & 0.23 & & \\
\hline \multirow{2}{*}{$\begin{array}{l}\text { Equation } 4.15 \text { (Hypothesis 2). Conditional total } \\
\text { effect of } \eta_{2} \text { on } \eta_{4}\end{array}$} & & & & & \\
\hline & 0.27 & 0.04 & 0.34 & 0.96 & 1.24 \\
\hline \multirow[t]{2}{*}{ conditioned on $\eta_{2}=$} & - & - & - & 0.90 & 2.90 \\
\hline & 2.10 & 1.10 & 0.10 & & \\
\hline \multirow{2}{*}{$\begin{array}{l}\text { Equation } 4.18 \text { (Hypothesis } 4 \text { ) Condit. non indirect } \\
\text { effect of } \eta_{l} \text { on } \eta_{4}\end{array}$} & & - & & & \\
\hline & 0.57 & 0.26 & 0.04 & 0.35 & 0.96 \\
\hline
\end{tabular}

Table 2 clearly shows how much the effect of each factor on performance could differ when the values of the other factor are varied. They can have negative effects for low values of innovation and of the interactive use of the budget while the magnitude of the effects can be considerable when the factors take on high values. The fact that negative effects are possible for certain values does not support hypotheses 2 and 4 .

\section{Discussion}

From the introduction, the reader is aware that the purpose of this paper is mainly methodological. The paper provides a modification and an extension of Jöreskog and Yang's approach for modeling interactions.

This paper started by referring to the need for handling measurement errors through SEM instead of using MRA for modeling interaction as marketing studies usually do. A re-analysis of the same data using MRA on summated rating scales yielded a $\mathrm{R}^{2}$ for performance equal to 0.29 , about the half of the $R^{2}$ obtained in this paper. This is the result of measurement error attenuation.

Given that most analyses refer only to covariances (the mean structure being unnecessary to identify the relevant regression slope parameters) we propose a modification of Jöreskog and Yang's SEM strategy by centering the original indicators prior to computing the interaction term, and then centering the interaction term as well. In this way we avoid collinearity while parsimony increases in the single indicator approach. As a consequence, the additional constraints involved in the measurement model's structure are simplified, which dramatically reduces the software set up. The only disadvantage may be a small loss in precision of the estimates due to the loss of one degree of freedom as a result of the constraints imposed on the mean structure. This drawback could not be observed for our data, for which standard errors were even $2 \%$ lower on average when analysing only the covariance structure. Changes in the point estimates were also very minor. 
Finally, the crucial question this paper tries to answer is: if one of the strengths of SEM is modeling indirect and direct effects together, why should interaction always be modeled only with direct effects? The paper specifies a simultaneous equation system to jointly estimate direct, indirect and interaction effects in a single step and in the same model using full information estimation procedures. The covariance structure involved by the simultaneous equation system is also set forth. In addition, the paper proposes an original, very straightforward interpretation of the results, analysing the total effects in terms of direct, indirect and interaction effects (Equations 4.14 to 4.18 , Figure 4 and Table 2). Though only one very simple case has been presented, the potential for generalizations is large. Only the variances and covariances of both variables that interact have to be expressed as a function of model parameters by means of path analysis.

\section{Acknowledgements}

We appreciate the comments by Fang Yang and Albert Satorra to previous versions of this paper. An earlier version was presented at the second EURAM annual conference on innovative research in management, Stockholm, May 9-11, 2002 .

\section{References}

[1] Abernethy, M. and Brownell, P. (1999): The role of budgets in organization facing strategic change: an exploratory study. Accounting, Organizations and Society, 24, 189-204

[2] Aiken, L.S. and West, S.G. (1991): Multiple Regression: Testing and Interpreting Interactions. Newbury Park, CA: Sage Publications.

[3] Algina, J. and Moulder, B.C. (2001): A note on estimating the Jöreskog Yang model for latent variable interaction using LISREL 8.3. Structural Equation Modeling, 8, 40-52.

[4] Anderson, T.W. (1984): An Introduction to Multivariate Statistical Analysis (2nd ed.). New York: Wiley.

[5] Ashok, K. Dillion, W.R., and Yuan, S. (2002): Extending discrete choice models to incorporate attitudinal and other latent variables. Journal of Marketing Research, 39, 31-46.

[6] Bagozzi, R.P. and Yi, Y. (1989): On the use of structural equation models in experimental designs. Journal of Marketing Research, 26, 271-284

[7] Batista-Foguet, J.M., Coenders, G., and Artés, M. (2001): Using structural equation models to evaluate the magnitude of measurement error in blood Pressure. Statistics in Medicine, 20, 2351-2368. 
[8] Bisbe, J. (2002): The Interactive Use of Management Control Systems on Product-Innovating Firms. Unpublished PhD dissertation ESADE. University Ramon Llull.

[9] Bollen, K.A. and Paxton, P. (1998): Interactions of latent variables in structural equation models. Structural Equation Modeling, 5, 267-293.

[10] Boomsma A. and Hoogland, J.J (2001): The robustness of LISREL modeling revisited. In R. Cudeck, S. du Toit and D. Sörbom (Eds.): Structural Equation Modeling: Present and Future. Lincolnwood, IL: Scientific Software International, 139-168.

[11] Browne, M.W. (1984): Asymptotically distribution-free methods for the analysis of covariance structures. British Journal of Mathematical and Statistical Psychology, 37, 62-83.

[12] Fouladi, R.T. (2000): Performance of modified test statistics in covariance and correlation structure analysis under conditions of multivariate non-normality. Structural Equation Modeling, 7, 356-410.

[13] Govindarajan, V. (1984): Appropriateness of accounting data in performance evaluation: an empirical examination of environmental uncertainty as an intervening variable. Accounting, Organizations and Society, 9, 33-48.

[14] Govindarajan, V. (1988): A contingency approach to strategy implementation at the business-unit level: integrating administrative mechanisms with strategy. Academy of Management Journal, 31, 828-853.

[15] Hartmann, F.G.H. and Moers, F. (1999): Testing contingency hypothesis in budgetarian research: and evaluation of the use of moderate regression analysis. Accounting, Organizations and Society, 24, 291-315.

[16] Homer, P.M. (1990): The mediating role of attitude toward the ad: some addition. Journal of Marketing Research, 27, 78-96.

[17] Irwin, J.R. and McClelland, G.H. (2001): Misleading heuristics and moderate regression models. Journal of Marketing Research, 38, 100-109.

[18] Jaccard, J. and Wan, C.K. (1995): Measurement error in the analysis of interaction effects between continuous predictors using multiple regression. Psychological Bulletin, 117, 348-357.

[19] Jaccard, J. and Wan, C.K. (1996): LISREL Approaches to Interaction Effects in Multiple Regression. Thousand Oaks, CA: Sage Publications.

[20] Jaccard, J., Turrisi, R., and Wan, C. K.(1990): Interaction Effects in Multiple Regression. Newbury Park, CA: Sage Publications.

[21] Jöreskog, K. (1973): A general method for estimating a linear structural equation system. In A.S. Goldberger and O. D. Duncan (Eds.): Structural Equation Models in the Social Sciences. New York: Seminar Press, 85-112.

[22] Jöreskog, K. (1998): Interactions and nonlinear modelling: issues and approaches. In R.E. Schumacker and G. A. Marcoulides (Eds.): Interactions and Nonlinear Effects in Structural Equation Models. Mahwah, NJ: Lawrence Erlbaum, 239-250. 
[23] Jöreskog, K. and Yang, F. (1996): Nonlinear structural equation models: the KennyJudd model with interaction effects. In G.A. Marcoulides and R.E. Schumacker (Eds.): Advanced Structural Equation Modeling. Mahwah, NJ: Lawrence Erlbaum, 57-89.

[24] Kenny, D.A. and Judd, C. M. (1994): Estimating the non-linear and interactive effects of latent variables. Psychological Bulletin, 96, 201-210.

[25] Li, F., Harmer, P., Duncan, T.E., Duncan, S.C., Acock, A., and Boles, S. (1998): Approaches to testing interactions effects using structural equation modeling methodology. Multivariate Behavioral Research, 33, 1-39.

[26] Li, H. and Atuahene-Gima, K. (2001): Product innovation strategy and the performance of new technology ventures in China. Academy of Management Journal, 44, 1123-1134.

[27] Likert, R. (1932): A technique for the measurement of attitudes. Archives of Psychology, 22, 1-55.

[28] Lomax, R.G. (1983): A guide to multi-sample structural equation modeling. Behavior Research Methods and Instrumentation, 15, 580-584.

[29] Moulder, B.C. and Algina, J. (2002): Comparison of methods for estimating and testing latent variables interactions. Structural Equation Modeling, 9, 1-19.

[30] Muthén, B. and Kaplan, D. (1992): A comparison of some methodologies for the factor analysis of non-normal Likert variables. A note on the size of the model. British Journal of Mathematical and Statistical Psychology, 45, 19-30.

[31] Ping, R. A. (1995): A parsimonious estimating technique for interaction and quadratic latent variables. Journal of Marketing Research, 32, 336-347.

[32] Ping, R. A. (1996): Latent variable interaction and quadratic effect estimation: A two step technique using structural equation analysis. Psychological Bulletin, 119, 166175.

[33] Ridgeon, E.E., Schumacker R.E. and Wothke, W. (1998): A comparative review of interaction and nonlinear effects modeling. In R.E. Schumacker and G.A. Marcoulides (Eds.): Interactions and Nonlinear Effects in Structural Equation Models. Mahwah, NJ: Lawrence Erlbaum, 1-16.

[34] Saris, W.E. and Satorra, A. (1988): Characteristics of structural equation models which affect the power of the likelihood ratio test. In W.E. Saris and I. N. Galhofer, (Eds.): Sociometric Research: Vol 2. Data Analysis. London: MacMillan, 220-236.

[35] Satorra, A. (1990): Robustness issues in structural equation modeling: a review of recent developments. Quality and Quantity, 24, 367-386.

[36] Satorra, A. (2001): Goodness of fit testing of structural equation models with multiple group data and nonnormality. In R. Cudeck, S. du Toit, and D. Sörbom (Eds.): Structural Equation Modeling: Present and Future. Lincolnwood, IL: Scientific Software International, 231-256. 
[37] Satorra, A. and Bentler, P.M. (1988): Scaling corrections for chi-square statistics in covariance structure analysis. ASA Proceedings of the Business and Economic Section, 308-313.

[38] Satorra, A. and Bentler, P.M. (1994): Corrections to test statistics and standard errors in covariance structure analysis. In A. von Eye and C. Clogg, (Eds): Latent Variables Analysis: Applications to Developmental Research. Thousand Oaks, CA: Sage publications, 399-419.

[39] Satorra, A. and Bentler, P. M. (2001): A scaled difference chi-square test statistic for moment structure analysis. Psychometrika, 66, 507-514.

[40] Schumacker, R.E. (2002): Latent variable interaction modeling. Structural Equation Modeling, 9, 40-54.

[41] Schumacker, R.E. and Marcoulides, G.A. (1998), Interactions and Nonlinear Effects in Structural Equation Models. Mahwah, NJ: Lawrence Erlbaum.

[42] Sethi, R., Smith, D.C., and Park, W. (2001): Cross-functional product development teams, creativity, and the innovativeness of new consumer products. Journal of Marketing Research, 38, 73-85.

[43] Simons, R. (1991): Strategic orientation and top management attention to control systems. Strategic Management Journal, 12, 49-62.

[44] Simons, R. (1995): Levers of control. Boston: Harvard Business School Press.

[45] Simons, R. (2000): Performance Measurement and Control Systems for Implementing Strategies. Upper Saddle River: Prentice Hall.

[46] Simpson, T. (1755): A letter to the right Honorable George Earl of Marclesfield, president of the Royal Society on the advantage of taking the mean of a number of observations in practical astronomy. Philosophical Transactions of the Royal Society, 4982-93.

[47] Spector, P.E. (1992): Summated Rating Scale Construction. An Introduction. Newbury Park, CA: Sage Publications.

[48] du Toit, M. and du Toit, S. (2001): Interactive LISREL, User's Guide, Chicago, IL: Scientific Software International.

[49] Yang Jonsson, F. (1998): Modeling nteraction and nonlinear effects in structural equation modeling: A step-by-step LISREL example. In R.E. Schumacker and G. A. Marcoulides (Eds.): Interactions and Nonlinear Effects in Structural Equation Models. Mahwah, NJ: Lawrence Erlbaum, 17-42.

[50] Yang Wallentin, F. and Jöreskog, K. (2001): Robust standard errors and chisquares for interaction models. In G. A. Marcoulides and R. E. Schumacker (Eds.): New Developments and Techniques in Structural Equation Modeling. Mahwah, NJ: Lawrence Erlbaum, 159-171. 


\section{Appendix}

Setup for modeling the interaction and indirect effects with LISREL 8.51

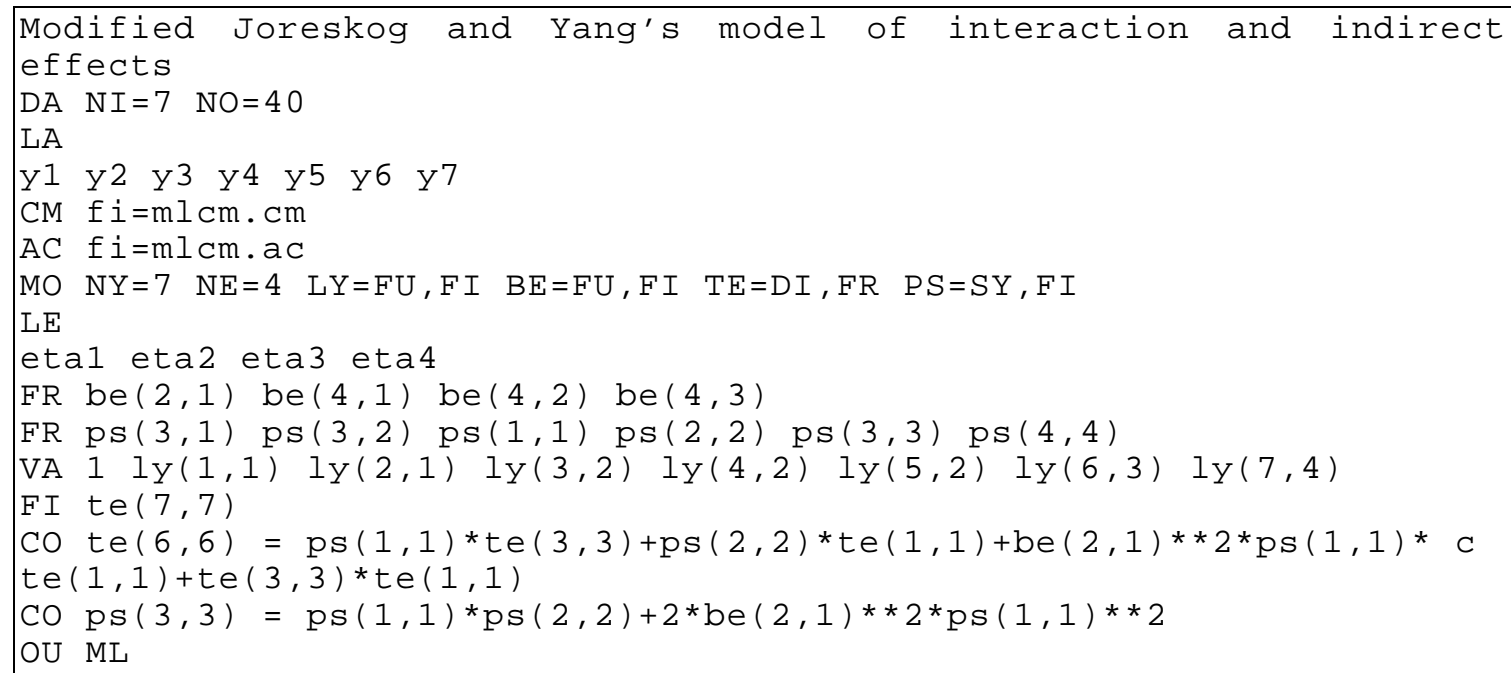

